\title{
tic\&société
}

Vol. 12, $\mathrm{N}^{\circ} 2$ | 2ème semestre 2018

Numérique et situations de handicap : les enjeux de l'accessibilité

\section{Rendre une ville intelligente-numérique accessible pour tous : le cas des personnes présentant une déficience intellectuelle}

Alejandro ROMERO-TORRES, Claude L. NORMAND et Dany LUSSIERDESROCHERS

\section{OpenEdition}

\section{Journals}

Édition électronique

URL : http://journals.openedition.org/ticetsociete/2657

DOI : 10.4000/ticetsociete.2657

Éditeur

Association ARTIC

Édition imprimée

Pagination : 45-73

\section{Référence électronique}

Alejandro ROMERO-TORRES, Claude L. NORMAND et Dany LUSSIER-DESROCHERS, « Rendre une ville intelligente-numérique accessible pour tous : le cas des personnes présentant une déficience intellectuelle », tic\&société [En ligne], Vol. 12, N 2 | 2ème semestre 2018, mis en ligne le 10 décembre 2018, consulté le 01 mai 2019. URL : http://journals.openedition.org/ticetsociete/2657 ; DOI : 10.4000/ticetsociete. 2657 
tic\&société - 12(2), 2018

\title{
Rendre une ville intelligente-numérique accessible pour tous : le cas des personnes présentant une déficience intellectuelle
}

\begin{abstract}
Alejandro ROMERO-TORRES
Alejandro Romero-Torres est professeur dans les programmes de $2^{\mathrm{e}}$ cycle en gestion de projet et en technologies de l'information à l'École de sciences de la gestion UQAM. II détient un doctorat en génie industriel de la Polytechnique de Montréal. Ses travaux portent sur l'adoption et la diffusion de nouvelles technologies, le rôle de l'utilisateur final dans le développement de systèmes technologiques et la participation des citoyens dans les villes intelligentes. Alejandro collabore comme chercheur associé à la Chaire en gestion de projets (UQAM), à l'Institut universitaire en déficience intellectuelle et en trouble du spectre de l'autisme (IU en DI-TSA) et au centre interuniversitaire de recherche et analyse des organisations (CIRANO). romero-torres.alejandro@uqam.ca
\end{abstract}

\section{Claude L. NORMAND}

Claude L. Normand est professeure agrégée au Département de psychoéducation et de psychologie à l'Université du Québec en Outaouais, Gatineau, Canada. Elle est d'abord reconnue pour ses travaux sur l'adaptation des parents dont les enfants ont des besoins particuliers et ses recherches portent généralement sur la participation sociale des personnes qui présentent une déficience intellectuelle (DI) ou un trouble du spectre de l'autisme (TSA) à tous les âges de la vie. Ses projets les plus récents portent sur la façon dont les TIC pourraient favoriser l'inclusion et la participation sociale des personnes qui présentent une DI ou un TSA dans le monde numérique, grâce entre autres aux médias sociaux (par ex. : Facebook, Snapchat, Instagram). Ceci l'a amenée à constater que l'accessibilité numérique est loin d'être acquise pour les personnes qui présentent des incapacités, étant confrontées aux 
Rendre une ville intelligente-numérique accessible pour tous : le cas des personnes présentant une déficience intellectuelle

exigences financières, sensorimotrices, cognitives, techniques ou sociales des TIC. Claude.Normand@uqo.ca

\section{Dany LUSSIER-DESROCHERS}

Dany Lussier-Desrochers est professeur titulaire au Département de psychoéducation de l'Université du Québec à Trois-Rivières et directeur général du Centre de partage d'expertise en intervention technoclinique (CPEITC). Depuis quelques années, il accompagne certains services spécialisés des Centres intégrés de santé et de services sociaux (CISSS) et des Centres intégrés universitaires de santé et de services sociaux (CIUSSS) du Québec dans l'implantation de l'intervention technoclinique. II développe également une programmation de recherche axée sur la promotion de l'inclusion numérique des personnes présentant une déficience ou des incapacités. Dany.Lussier-Desrochers@uqtr.ca 


\author{
Alejandro ROMERO-TORRES, Claude L. NORMAND et \\ Dany LUSSIER-DESROCHERS
}

\title{
Rendre une ville intelligente-numérique accessible pour tous : le cas des personnes présentant une déficience intellectuelle
}

Résumé : La ville intelligente-numérique vise à développer et intégrer une infrastructure basée sur les technologies de l'information et de la communication pour améliorer la qualité de vie de l'ensemble des citoyens et pour obtenir plus rapidement des informations et des services gouvernementaux. Toutefois, l'arrivée de cette nouvelle ère entraîne de nouveaux enjeux, dont celui de la fracture numérique. Basé sur le contexte et les enjeux des personnes ayant une déficience intellectuelle, cet article présente un modèle d'engrenage de l'accessibilité au monde numérique, des pistes de solution de même que trois recommandations pour développer des partenariats entre les citoyens et les institutions permettant de créer une communauté intelligente-numérique.

Mots-clés : ville intelligente, accessibilité, déficience intellectuelle, citoyen.

Abstract: Smart and digital cities aim to develop and integrate an information and communication technology-based infrastructure to improve their citizens' quality of life and accelerate access to information and public services. However, this digital era brings new challenges, including a digital divide between certain segments of the population. Based on the context and challenges of people with intellectual disabilities, this article presents a model for enabling accessibility to digital services as well as three recommendations for developing partnerships between citizens and institutions in order to create an inclusive smart-digital community.

Keywords: smart city, accessibility, intellectual disability, citizen. 
Rendre une ville intelligente-numérique accessible pour tous : le cas des personnes présentant une déficience intellectuelle

Resumen: Una ciudad digital e inteligente tiene como objetivo desarrollar e integrar infraestructuras basadas en las tecnologías de la información y la comunicación de cara a mejorar la calidad de vida de todos los ciudadanos y permitirles acceder más rápidamente a la información y a los servicios gubernamentales. No obstante, la llegada de esta nueva etapa implica nuevos desafíos, y en especial el de la brecha digital. Basado en el contexto y en los desafíos de las personas con limitaciones intelectuales, este artículo, presenta un modelo de accesibilidad al mundo digital, ofrece pistas de solución y establece tres recomendaciones para el desarrollo de colaboraciones entre ciudadanos e instituciones que permitan crear una comunidad digital inteligente e inclusiva.

Palabras clave: ciudad inteligente, accesibilidad, limitaciones intelectuales, ciudadano. 


\section{Alejandro ROMERO-TORRES, Claude L. NORMAND et Dany LUSSIER-DESROCHERS}

\section{Introduction}

Au-delà des objectifs de marketing (Simard, 2015), le concept de ville intelligente-numérique (VIN) vise à développer et à intégrer une infrastructure basée sur les technologies de l'information et de la communication (TIC) pour améliorer la qualité de vie de l'ensemble des citoyens. En ce sens, la VIN traduit une évolution des pratiques où le citoyen n'est pas seulement un consommateur: c'est un producteur d'idées, d'engagement et d'inclusion sociale (de Lange et de Waal, 2015). À la suite du défi lancé aux secteurs public et privé par l'ex-président Bill Clinton en 2005 pour développer des services en s'appuyant sur des TIC de pointe, plusieurs villes, telles que Londres, Séoul, Barcelone et Amsterdam, se sont lancées dans cette aventure (RodriguezBolivar, 2015). Quelques années plus tard, des métropoles canadiennes ont aussi adopté des stratégies visant l'offre numérique de leurs services, le développement des solutions technologiques liées aux enjeux urbains, la mobilisation des citoyens en temps réel et l'accès à la vie démocratique (Gabrys, 2014). La VIN permet alors au citoyen d'obtenir plus rapidement des informations et des services gouvernementaux (CEFRIO, 2013). Ces initiatives s'inscrivent dans la foulée des résultats de plusieurs études démontrant l'influence des TIC sur le pouvoir d'agir des personnes et la promotion de la participation citoyenne égalitaire (Jenkins, 2006).

Toutefois, l'arrivée de cette nouvelle ère entraîne de nouveaux enjeux, dont celui de la fracture numérique (Helsper, 2012). Actuellement, certaines personnes ne peuvent pas contribuer à la société intelligente-numérique ni en tirer profit, car elles ne possèdent pas les outils (ordinateurs, tablettes numériques ou téléphones intelligents) ni les connaissances et les compétences nécessaires (notamment les compétences cognitives telles que le raisonnement, la planification ou la mémoire à court et à long terme) pour interagir avec le numérique (Wei et Hindman, 2011). C'est le cas, notamment, d'une bonne proportion de personnes présentant des limitations cognitives (Wei et al., 2011). En effet, 
Rendre une ville intelligente-numérique accessible pour tous : le cas des personnes présentant une déficience intellectuelle

ces dernières doivent interagir avec un environnement numérique commun à l'ensemble de la société qui s'avère inadapté à leurs besoins (Dagenais, Poirier et Quidot, 2012). Elles doivent en outre développer des compétences numériques spécialisées, ce qui n'est pas une tâche facile. Cette situation est d'autant plus problématique pour les personnes présentant une déficience intellectuelle (DI) qui, selon plusieurs auteurs (dont Hoppestad, 2013), devraient constituer le groupe de référence pour la conception des outils technologiques au même titre que les personnes en fauteuil roulant représentent le groupe de référence lorsqu'il est question d'aménager l'accessibilité aux immeubles (Gossett et al., 2009).

Cet article a trois objectifs: 1) comprendre les enjeux d'exclusion liés au développement de la VIN ; 2) identifier les exigences d'accessibilité pour une société intelligente-numérique inclusive; et 3) proposer des pistes d'action. Cet article est structuré en cinq parties. La première et la deuxième partie décrivent d'abord spécifiquement les caractéristiques d'une ville intelligente-numérique et la double exclusion observée dans ces initiatives. Ensuite, nous présentons les exigences d'accessibilité numérique universelle et les étapes pour créer une communauté intelligente-numérique inclusive. La dernière section présente enfin, en guise de conclusion, des pistes d'action possibles pour rendre la VIN plus accessible pour tous.

\section{Une ville intelligente-numérique}

Selon les estimations de l'ONU, d'ici 2050, $70-75 \%$ de la population mondiale vivra en ville (Caragliu, Del Bo et Nijkamp, 2011). Sans aucun doute, ce taux d'urbanisation sans précédent est un véritable facteur de réussite pour le progrès économique et social de la planète, mais il représente en même temps, un énorme défi pour les municipalités. Elles sont confrontées à rationaliser leurs ressources, telles que l'infrastructure, l'eau, l'énergie et le transport public. Compte tenu de cette situation, les gouvernements sont obligés de changer le modèle de gestion de leurs villes, de sorte que les conséquences de cette croissance soient atténuées. 


\section{Alejandro ROMERO-TORRES, Claude L. NORMAND et Dany LUSSIER-DESROCHERS}

Une VIN est définie comme une zone géographique qui combine les TIC et la technologie Web 2.0 avec d'autres efforts organisationnels pour améliorer sa durabilité et son habitabilité, dématérialiser et accélérer les processus bureaucratiques et identifier des solutions novatrices aux enjeux municipaux (Toppeta, 2010). Caragliu, Del Bo et Nijkamp (2011) ajoutent qu'une VIN est caractérisée par les investissements en capital humain, en capital social et en infrastructures, soit traditionnelles (transport) et modernes (TIC), pour assurer la croissance économique, le développement durable et la qualité de vie des citoyens. Nam et Pardo (2011) affirment que l'objectif de la VIN est de créer un environnement propice à l'échange de l'information, la collaboration et l'interopérabilité en utilisant les innovations technologiques pour améliorer les services et en créer des nouveaux. Par contre, les TIC ne peuvent pas rendre par ellesmêmes un service intelligent. Pour ce faire, il est nécessaire de développer une infrastructure où la participation et la collaboration entre citoyens, fonctionnaires, élus, fournisseurs, partenaires et entreprises soient mises en valeur (Caragliu, Del Bo et Nijkamp, 2011).

II existe différents modèles qui permettent de caractériser une VIN, mais le plus utilisé dans la littérature est celui de Cohen (2012) (voir Figure1). Ce modèle identifie six dimensions clés avec 18 facteurs permettant d'évaluer l'infrastructure des villes. Ces dimensions sont: environnement, citoyens, gouvernement, mobilité, milieu de vie et économie. Cohen accompagne ces dimensions et ces facteurs par 100 indicateurs différents qui permettent d'évaluer et de comparer les villes voulant intégrer des projets intelligents et numériques. 
Rendre une ville intelligente-numérique accessible pour tous : le cas des personnes présentant une déficience intellectuelle

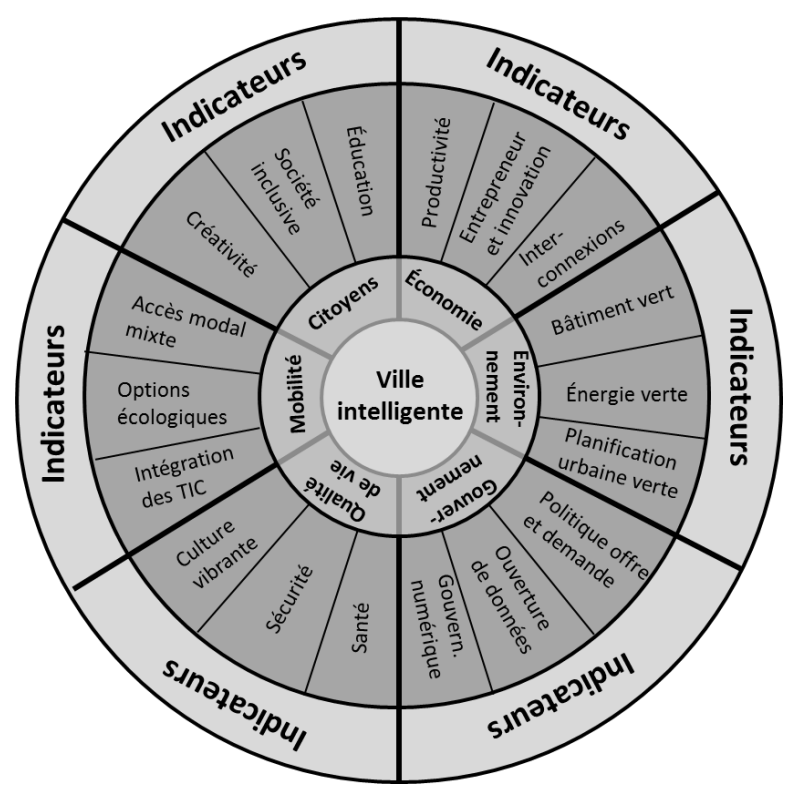

Figure 1. Une ville intelligente de Cohen (traduction libre, 2012)

La VIN a entraîné l'émergence du concept de citoyen intelligentnumérique (en anglais, smart citizen), dans lequel il est mobilisé pour participer à la création d'espaces et de services publics (Lee et Lee, 2014), ainsi qu'à la gouvernance de sa municipalité (Caragliu et Del Bo, 2015). Historiquement, les citoyens ont rarement été impliqués dans la conception ou l'exécution de projets municipaux. Ceux-ci sont généralement mis en œuvre et gérés par des experts en TIC qui ont une vague idée des défis auxquels font face les communautés locales (Sadoway et Shekhar, 2014). Les outils numériques tels que les sites Web 2.0 et les applications pour les tablettes ou les téléphones intelligents permettent aux citoyens d'établir un contact direct avec les fonctionnaires pour leur transmettre des données sur l'état actuel des infrastructures municipales. Ces outils sont utilisés pour suggérer des idées d'amélioration (Norris et Moon, 2005). Par contre, les stratégies choisies pour définir, développer et implanter les services numériques ont été modulées selon les besoins des organismes commanditaires (par ex., les municipalités) (Jaeger et Bertot, 2010), excluant les citoyens (Chen et Dimitrova, 2008). 


\section{Alejandro ROMERO-TORRES, Claude L. NORMAND et Dany LUSSIER-DESROCHERS}

\section{Les défis de l'inclusion}

II est inapproprié de considérer que tous les citoyens ont accès aux technologies numériques. Selon le PEW Reserach Center (2018), $65 \%$ des Américains ont une connexion par l'intermédiaire d'un fournisseur de haut débit, $20 \%$ l'ont uniquement par l'intermédiaire de leur téléphone intelligent et $15 \%$ ne possèdent pas de connexion à l'Internet ou à des interfaces numériques. Par contre, il y a alors une bonne proportion des individus qui n'ont pas les outils pour consommer ou produire des données numériques. De plus, des personnes ayant des limitations cognitives (par exemple, des problèmes de mémoire à court et à long terme, de déduction ou de planification) doivent interagir avec un environnement numérique commun à l'ensemble des citoyens, qui n'a pas été adapté à leurs besoins (Dagenais, Poirier et Quidot, 2012). Les services intelligents-numériques ne tiennent pas compte des compétences et des connaissances ni du contexte d'utilisation de ces individus. Par exemple, soit il y a trop d'informations, soit le fonctionnement n'est pas logique ou le texte est trop petit. Cet écart se traduit plus précisément par un sentiment d'exclusion nommé "fracture numérique " (Vodoz, 2010).

Pour les citoyens présentant une $\mathrm{DI}$, l'exclusion numérique se conjugue alors à une exclusion sociale (Helsper, 2012). Toutefois, aucune initiative impliquant des personnes handicapées, y compris celles présentant des limitations cognitives, n'a été documentée. Ceci est en cohérence avec les travaux de Schejter, Ben Harush et Tirosh (2015), qui ont démontré que la fracture numérique est non seulement un élément de séparation technologique, mais qu'elle génère en plus une exclusion sociale. Ce phénomène va à l'encontre des politiques sociales et des cadres législatifs actuellement en vigueur dans plusieurs pays industrialisés qui soulignent l'importance d'assurer une participation pleine et entière de ces personnes dans toutes les dimensions de la vie sociale (PCPID, 2015). 
Rendre une ville intelligente-numérique accessible pour tous : le cas des personnes présentant une déficience intellectuelle

\section{Les exigences de l'accessibilité}

Pour concevoir des services intelligents-numériques inclusifs, il faut d'abord identifier quels sont les obstacles à cette inclusion. En d'autres termes, il faut identifier les exigences du monde numérique que les citoyens doivent rencontrer pour profiter de la VIN. Une recension exhaustive des écrits a fait ressortir cinq exigences spécifiques qui ont creusé un fossé numérique entre les personnes qui présentent une DI et la population branchée, soit 1) l'accès aux TIC, 2) les exigences sensorimotrices, 3) cognitives, 4) techniques et 5) les codes et les conventions sociales (LussierDesrochers et al., 2016 ; Lussier-Desrochers et al., 2017). Ces exigences que les citoyens doivent rencontrer pour profiter de la VIN peuvent être représentées graphiquement sous forme d'un engrenage, où chacune d'elles interagit avec les autres (Figure 2). Au centre se trouvent les ressources de la personne et celles de son environnement, qui permettent de faire tourner la roue, et donc d'avancer sur le continuum de la participation sociale numérique.

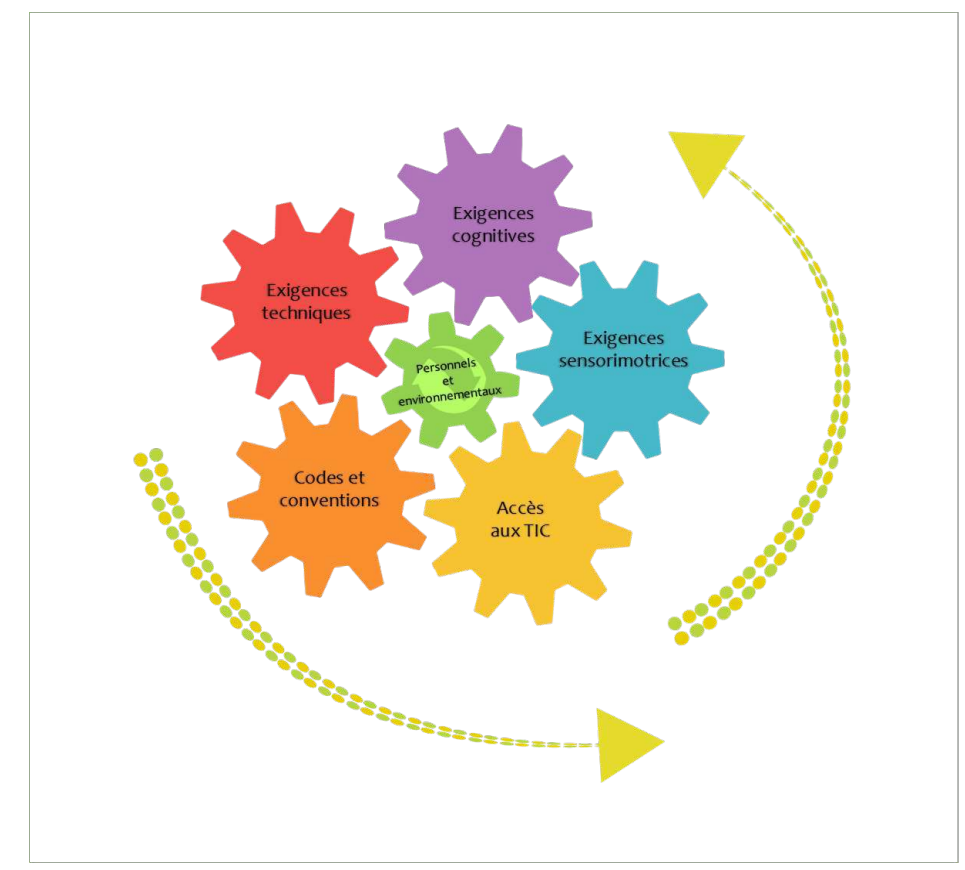

Figure 2. Engrenage de l'accessibilité au monde numérique. 


\section{Alejandro ROMERO-TORRES, Claude L. NORMAND et Dany LUSSIER-DESROCHERS}

Les paragraphes qui suivent définissent les exigences de l'inclusion numérique, les ressources personnelles nécessaires, de même que les ressources à mettre en place dans l'environnement de l'utilisateur des TIC pour assurer sa participation dans le monde numérique.

\subsection{L'accès aux TIC}

Comme mentionné précédemment, une proportion assez importante des citoyens européens de plus de 50 ans, ou ceux présentant une limitation cognitive, ne possède pas son propre ordinateur, sa tablette ou son téléphone intelligent. Par ailleurs, on estime à près de $15 \%$ la proportion des ménages nord-américains qui n'ont pas d'accès Internet (Eurostat, 2016). Or pour bénéficier des contenus et des services de la VIN, les citoyens doivent nécessairement passer par l'intermédiaire des TIC connectées à un réseau. Cela nécessite un certain investissement financier pour l'achat de matériel (ordinateur, téléphone intelligent, tablette numérique, routeur) et de logiciels ou applications, auquel s'ajoute l'abonnement à un fournisseur de réseau.

On constate que les ressources financières insuffisantes de certains citoyens constituent un obstacle majeur à l'accessibilité alors même que la population avec des limitations cognitives ou autres incapacités est particulièrement touchée par la pauvreté (Groce et al., 2011). Si des adaptations, des applications ou des équipements spécialisés sont nécessaires pour rendre les TIC accessibles et utilisables par ces citoyens, des coûts additionnels sont à prévoir, en deçà de ce qu'il en coûte "normalement » au reste de la population.

Néanmoins, l'accès aux TIC peut être facilité par la société de diverses manières. À son arrivée dans nos foyers, l'ordinateur de table a longtemps été partagé entre tous les membres de la famille ou de la résidence. Plus récemment, les TIC sont devenues de plus en plus petites, portatives et individualisées, ce qui en restreint l'accès «communautaire »: partager un téléphone intelligent entre tous les membres d'une même famille paraîtrait farfelu de nos jours. 
Rendre une ville intelligente-numérique accessible pour tous : le cas des personnes présentant une déficience intellectuelle

Des organismes communautaires peuvent offrir de l'équipement usagé gratuitement, ou à peu de frais. Malheureusement, dans plusieurs cas de dons de matériel informatique, les appareils sont désuets et sujets à des problèmes techniques (Dagenais, Poirier et Quidot, 2012). Ceci pourrait alors mener à une sous-utilisation, sinon l'abandon, de la technologie.

Des programmes d'accès (par ex., bourses, subventions) gouvernementaux ou privés permettent à certaines personnes avec des limitations fonctionnelles qui se qualifient d'acquérir leur propre appareil. Certes, ces initiatives permettront l'achat du " hardware », mais elles ne vont pas couvrir les coûts afférents tels le branchement à un réseau Internet, un boîtier de protection, l'achat d'applications ou de périphériques spécialisés, ni bien sûr l'entretien. En somme, le coût total de possession (incluant les frais directs et indirects, tels que la connexion aux réseaux, les logiciels de sécurité ou la maintenance) d'une tablette numérique est souvent le double du coût initial d'achat de la tablette ellemême (Morphy, 2010).

Reste l'option du prêt. De plus en plus de bibliothèques municipales offrent un service d'emprunt de tablettes électroniques, d'une durée et en quantités limitées. Par contre, ce mode d'emprunt pourrait comporter certains risques concernant la protection de la vie privée ou d'informations personnelles. De plus, lorsque des limitations motrices s'ajoutent aux limitations cognitives d'un certain segment de la population (Dagenais, Poirier et Quidot, 2012; Michel, Masson et Sperandio, 2006), les appareils sont à risque d'être échappés. L'absence de périphériques adaptés peut aussi réduire l'utilité des emprunts de TIC standards. À ces défis s'ajoute la complexité du déplacement requis pour effectuer cet emprunt à la bibliothèque. Au Québec, des organismes gouvernementaux ont également créé des programmes de prêt de matériel (AQETA, 2016), mais une enquête a révélé que ces derniers ne semblent pas en mesure de répondre adéquatement à l'ensemble des besoins des personnes qui présentent une DI (Lussier-Desrochers, Caouette et GodinTremblay, 2016), sans compter qu'ils ne règlent en rien le besoin d'un accès à un réseau Internet. Bref, la dimension de l'accès physique à des TIC adaptées aux capacités et aux besoins 


\section{Alejandro ROMERO-TORRES, Claude L. NORMAND et Dany LUSSIER-DESROCHERS}

particuliers, et branchées sur le Web, des personnes présentant une DI constitue une barrière à l'inclusion numérique.

\subsection{Les exigences sensori-motrices}

Une seconde dimension de l'accessibilité à la VIN concerne les habiletés sensori-motrices (SM) du citoyen en contrepoids aux exigences des dispositifs numériques. En effet, l'ergonomie des TIC standards requiert un minimum d'aptitudes sur les plans sensoriels (principalement tactile, visuel, auditif et proprioceptif) et moteurs (motricité fine et globale) (Dagenais, Poirier et Quidot, 2012).

La présence concomitante de la DI et des limitations SM a des répercussions directes sur la réalisation d'actions élémentaires nécessaires pour naviguer en ligne. Par exemple, le manque de coordination, de préhension ou de dextérité des membres supérieurs peut entraver l'utilisation du clavier et de la souris, surtout si les appareils sont petits (Dagenais, Poirier et Quidot, 2012). De plus, la navigation sur le Web oblige les utilisateurs à réagir rapidement, ou dans un temps limité. Des contraintes motrices peuvent donc rendre la réalisation de certaines activités difficiles en raison des délais restreints (comme remplir un formulaire, faire un achat en ligne, etc.) (Dagenais, Poirier et Quidot, 2012). Par ailleurs, les personnes qui présentent une DI peuvent aussi avoir des limitations sensorielles, notamment visuelles et auditives, qui augmentent le niveau de difficulté de la navigation sur le Web (Dagenais, Poirier et Quidot, 2012). Un nombre croissant de sites Internet utilisent le multimédia comme mode de transmission de l'information, ce qui entrave leur accessibilité pour les personnes présentant des limitations visuelles et auditives. Selon Michel, Masson et Sperandio (2006), près de $80 \%$ à $90 \%$ des sites Internet ne seraient pas navigables par les personnes présentant ce type de difficultés.

Toutefois, le développement de périphériques et des logiciels spécialisés démontre qu'il est possible d'adapter les TIC aux besoins spécifiques des personnes qui présentent des limitations SM (Danial-Saad, Weiss et Schreuer, 2012). Actuellement, plusieurs périphériques sont disponibles sur le marché, tels que 
Rendre une ville intelligente-numérique accessible pour tous : le cas des personnes présentant une déficience intellectuelle

des souris adaptées (Danial-Saad, Weiss et Schreuer, 2012), des claviers surdimensionnés (Abilities Expo, 2015), des écrans tactiles (Stephenson et Limbrick, 2015), des logiciels permettant de faire fonctionner un ordinateur à l'aide des mouvements pupillaires (Dube et Wilkinson, 2014) et des systèmes de synthèse ou de reconnaissance vocale. Ce type de périphériques améliore l'accessibilité de la VIN pour les personnes présentant certaines limitations SM (Raghavendra et al., 2013).

\subsection{Les exigences cognitives}

Dans son analyse de la société numérique, Compiègne (2011) affirme que les capacités cognitives initiales des personnes restent déterminantes et discriminantes pour assurer une utilisation efficiente des technologies numériques. De fait, les technologies et le contenu numérique exigent la mobilisation de plusieurs fonctions cognitives, comme le raisonnement inductif, les habiletés de résolution de problèmes, la mémoire à court et à long terme, le raisonnement, la planification, la réflexion et la déduction (Dagenais, Poirier et Quidot, 2012 ; Lussier-Desrochers et al., 2011 ; Rivas-Costas et al., 2014). Or la déficience intellectuelle se définit entre autres par des limitations dans chacune de ces sphères. II en résulte des difficultés à comprendre le fonctionnement des appareils, à décoder des symboles, à entrer une requête dans un moteur de recherche, à sélectionner le contenu pertinent parmi les propositions offertes sur le Web, ou encore à comprendre l'information qui s'y trouve (Danielsson et al., 2012). Williams et Nicholas (2006) ont de plus observé un usage inefficace de la souris d'ordinateur (par ex., cliquer au mauvais endroit, ou trop souvent) par des personnes ayant des troubles d'apprentissage, même si ces dernières possèdent les habiletés motrices pour manipuler la souris (par ex., déplacer le curseur vers le bon endroit). Aussi, l'impulsivité caractéristique de plusieurs personnes avec un DI les amène à réagir précipitamment et à sélectionner la première option par défaut, ce qui représente un obstacle important lorsqu'il s'agit de trouver de l'information pertinente sur le Web (Lussier-Desrochers et al., 2011 ; Williams et Nicholas, 2006). 


\section{Alejandro ROMERO-TORRES, Claude L. NORMAND et Dany LUSSIER-DESROCHERS}

On ne saurait par ailleurs négliger la prévalence des compétences en lecture et en écriture nécessaires, de préférence en anglais, à la navigation sur le Web (Rivas-Costas et al., 2014), sans compter que la lecture des hypertextes exigerait plus d'efforts cognitifs que la lecture de textes en version papier (Chen, 2010).

La quantité, la variété et le fractionnement des informations que l'on peut trouver en ligne ont l'effet paradoxal de complexifier l'accès à l'information (Bunning et al., 2013). Wong et ses collaborateurs (2009) ont d'ailleurs démontré que plus le nombre d'étapes pour effectuer une opération à l'aide de TIC est élevé, plus les difficultés rencontrées seront grandes pour les personnes qui présentent des limitations cognitives. Enfin, même le design des TIC, comme la présence d'un même bouton pour ouvrir et fermer les appareils, peut créer de la confusion (Williams et Nicholas, 2006).

De nombreuses solutions peuvent être déployées dans l'environnement de la personne pour l'aider à répondre aux exigences cognitives des TIC. Par contre, la littérature scientifique ne nous permet pas à l'heure actuelle de déterminer lesquelles privilégier. Une solution simple, économique et disponible consiste à ajuster des paramètres du panneau de contrôle de l'ordinateur, notamment en modifiant le réglage de la souris (Rivas-Costas et al., 2014). Cependant, l'ajustement de ces paramètres s'avère insuffisant pour répondre aux multiples besoins des utilisateurs avec des limitations cognitives. Au cours des dernières années, des applications, des sites Internet et des navigateurs Web adaptés ou "simplifiés' ont été développés afin de diminuer la charge cognitive associée à l'utilisation des outils numériques (Perkins et LaMartin, 2012 ; Stock et al., 2006 ; Williams et Hennig, 2015 ; Wong et al., 2009). Enfin, certains croient que la solution serait d'appliquer des règles d'accessibilité universelle à la conception des TIC, des logiciels, des sites et des applications, permettant ainsi aux personnes en situation de handicap d'avoir accès au même environnement numérique que le reste de la population (Karreman, van der Geest et Buursink, 2007). Malheureusement, ces règles sont encore très peu considérées par les concepteurs (Bunning et al., 2010 ; Kennedy, Evans et Thomas, 2011). Serait-ce parce qu'elles sont beaucoup trop 
Rendre une ville intelligente-numérique accessible pour tous : le cas des personnes présentant une déficience intellectuelle

nombreuses? «Par exemple, on retrouve 944 recommandations dans le recueil de Smith et Mosier (1986) et plus de 3000 dans le recueil de Vanderdonckt (1995)" (Brangier, Hammes-Adelé et Bastien 2009, p. 4). Pour contourner le problème, on propose que les personnes qui présentent une DI constituent le groupe de référence pour la conception des outils technologiques étant donné leurs importantes difficultés cognitives (Hoppestad, 2013; Lussier-Desrochers et al., 2017).

\subsection{Les exigences techniques}

L'efficacité et la pérennité du matériel informatique nécessitent de prévenir les risques pouvant mettre en péril son bon fonctionnement, ou d'avoir assez de compétences techniques ou de ressources dans son réseau pour se dépanner lors d'une mise en échec. Certaines technologies, notamment celles branchées à l'Internet, exposent le matériel informatique de l'utilisateur à des virus, des logiciels espions, des chevaux de Troie ou d'autres programmes malveillants pouvant, si des dispositions préventives ne sont pas prises en compte (par ex., logiciel antivirus), endommager le matériel informatique de l'utilisateur et ainsi nécessiter la réparation ou même le remplacement de certaines composantes (Newman, 2009). Peu d'études se sont penchées spécifiquement sur les difficultés techniques rencontrées par les internautes qui présentent une DI. Néanmoins, une étude québécoise rapporte que certains participants auraient contracté des virus informatiques qui ont affecté le fonctionnement de leur ordinateur (Lussier-Desrochers et al., 2011). L'interdépendance des compétences techniques et des fonctions cognitives des personnes avec une DI expliquerait qu'ils ont rarement les connaissances ou le savoir-faire nécessaires pour résoudre seuls les défis techniques qu'ils rencontrent (Dagenais, Poirier et Quidot, 2012). En effet, l'utilisation des TIC nécessite périodiquement la résolution de problèmes techniques tels que la connexion aux réseaux publics ou privés, l'installation de nouveaux périphériques, la mise à jour d'applications ou de dispositifs et la réparation de pannes logicielles (Perriault, 2006). Ces actions élémentaires (techniques) peuvent rapidement devenir complexes (cognitivement) pour les personnes présentant une DI. Elles 


\section{Alejandro ROMERO-TORRES, Claude L. NORMAND et Dany LUSSIER-DESROCHERS}

devront alors aller chercher de l'aide chez d'autres utilisateurs, sur des sites Internet (souvent peu compréhensibles) dédiés à la résolution de problèmes techniques, ou chez des fournisseurs de services. Cette dernière solution est d'autant plus préoccupante en raison des ressources financières limitées des personnes présentant une DI qui réduisent leur accès à de tels services.

La prévention de défaillances techniques serait donc à privilégier. La configuration sécuritaire des appareils, la sécurisation du réseau sans-fil, l'installation d'un logiciel antivirus, la configuration du pare-feu et la mise à jour du système d'exploitation et des logiciels sont autant d'actions concrètes à réaliser (HabiloMédias, 2015). Toutefois, ces démarches sont souvent complexes sur le plan cognitif (Dagenais, Poirier et Quinot, 2012) et nécessitent un soutien extérieur.

\subsection{Les codes et les conventions sociales}

De nouvelles conventions et règles d'interactions sociales ont émergé avec la venue des TIC, qui permettent de communiquer de manière anonyme, à un auditoire innombrable d'inconnus et en temps différé, entre autres. Pour le destinataire, la prudence est de mise face à certaines formes de sollicitation. Une bonne compréhension de ces codes fait appel à des habiletés adaptatives conceptuelles (par ex. : abstraction, raisonnement) et sociales qui sont limitées avec le diagnostic de DI (Dagenais, Poirier et Quinot, 2012). Sans la compréhension de ces règles, l'utilisateur peut s'exposer à de nouvelles formes de victimisation en ligne (par ex. : sollicitation sexuelle en ligne, vol d'identité, achats impulsifs, harcèlement, exposition à des contenus non désirés) (Holtfreter et al., 2015). Elles peuvent aller jusqu'à mettre leur santé et leur sécurité en danger, par exemple en acceptant de rencontrer et d'avoir des relations sexuelles non protégées avec des inconnus trouvés sur le Web (Sallafranque St-Louis et Normand, 2017).

La crédulité, la tendance à l'acquiescement, le désir de plaire et l'impulsivité qui caractérisent de nombreuses personnes présentant une $\mathrm{DI}$ les mettent à risque de hameçonnage et d'escroquerie (Buijs et al., 2016 ; Holtfreter et al., 2015). De plus, il 
Rendre une ville intelligente-numérique accessible pour tous : le cas des personnes présentant une déficience intellectuelle

semble que ces dernières aient tendance à accepter d'emblée les propositions qui leur sont faites sur Internet par le biais des fenêtres contextuelles (Lussier-Desrochers et al., 2011). Des programmes d'éducation et du soutien individualisés doivent donc être développés (Dowdell, Burgess et Flores, 2011 ; Raghavendra et al., 2013). Par exemple, afin de favoriser l'utilisation sécuritaire des sites de réseaux sociaux, l'organisation Autism Speaks a émis, en 2011, dix recommandations destinées aux adolescents et aux jeunes adultes autistes, qui sont tout à fait pertinentes pour toute personne présentant des limitations cognitives et sociales. Parmi celles-ci, on retrouve notamment des mises en garde quant à la divulgation des données personnelles (adresse du domicile, numéro de téléphone, numéro de compte bancaire, photos), au contrôle des informations partagées (groupes privés, site Web avec un accès libre) et en rapport avec la rencontre en personne d'amis virtuels (se rencontrer dans un lieu public, informer des gens de son entourage, être accompagné). À cela s'ajoutent des solutions informatiques, politiques ou légales pour augmenter la sécurité des données ou celle des usagers (Charlotte, 2010 ; PCPID, 2015 ; United Nations Broadband Commission for Digital Development, 2015). Par exemple, Facebook a changé un de ses paramètres par défaut qui rendait certains affichages automatiquement publics. II est aussi intéressant de constater que des sites de rencontres font dorénavant des vérifications (par ex. : dossiers criminels) au sujet de leurs membres.

En somme, les ressources de la personne et celles de son environnement influencent les possibilités qui lui sont offertes de répondre aux cinq grandes exigences de la société du numérique et devenir un citoyen intelligent-numérique au sein de la VIN. Ce modèle (voir Figure 2) illustre les efforts à réaliser pour assurer l'accessibilité numérique. Le citoyen confronté à plusieurs obstacles de l'environnement numérique aura de la difficulté à faire avancer les engrenages sur la voie de la participation sociale. Le moyeu central devra donc exercer une plus grande force, en puisant davantage parmi les ressources personnelles ou environnementales. 


\section{Alejandro ROMERO-TORRES, Claude L. NORMAND et Dany LUSSIER-DESROCHERS}

\section{Construire des partenariats pour une communauté intelligente-numérique inclusive}

La mise en place de VIN peut être source de changements majeurs pour plusieurs de ses acteurs. En plus d'inclure des éléments hautement techniques, le développement des solutions numériques nécessite la mise en commun d'expertises en provenance de plusieurs disciplines. Bien que l'identification des acteurs clés soit nécessaire, elle n'est pas suffisante. La démarche doit être encadrée et structurée. Cette section de l'article présente la mise en place de partenariats comme une solution pour guider les futures démarches des communautés intelligentes numériques visant à rendre les VIN plus accessibles pour tous.

\subsection{Identifier les différentes parties prenantes et les enjeux auxquels elles seront confrontées}

Au cours des dernières années, les communautés ont vécu de nombreuses pressions systémiques les poussant à intégrer les technologies numériques à l'offre de services aux citoyens. De plus, on les incite à prendre en compte les besoins spécifiques des personnes en situation de handicap. Actuellement, l'intégration des technologies dans les villes constitue une innovation technologique, organisationnelle et sociale. Ce changement nécessite une réévaluation des services dispensés aux citoyens et une révision de la mission des différentes organisations offrant ces services. En plus d'apporter une certaine déstabilisation, cette situation peut susciter de la résistance au changement chez plusieurs acteurs clés. Dans ce contexte, leur consultation permettra d'identifier leurs perceptions relatives au changement qui s'amorce de même que les enjeux anticipés.

\subsection{Déterminer la vision commune unissant les parties prenantes}

Le réel défi de partenariat dans ces projets d'envergure consiste à faire converger les efforts de ces acteurs vers une vision consensuelle. Dans un contexte d'innovation, l'identification de la vision est essentielle. En effet, la vision constitue « une façon de 
Rendre une ville intelligente-numérique accessible pour tous : le cas des personnes présentant une déficience intellectuelle

voir, de concevoir un ensemble de choses complexes » (Le Grand Robert, 2016). La vision permet de bien circonscrire les actions menant à l'atteinte d'une cible commune. En somme, elle permet d'optimiser les efforts des parties prenantes. Ainsi, tout projet de partenariat d'une telle envergure devrait s'appuyer sur une vision reconnue et partagée. Plus concrètement, la vision devrait situer précisément la trajectoire permettant de passer de la situation actuelle à la situation souhaitée.

\subsection{Mettre en place un mécanisme de coordination et identifier des porteurs de l'innovation}

Le projet de VIN accessibles doit être porté par les membres de la communauté. Ces derniers s'engagent à concrétiser la vision développée par l'ensemble des parties prenantes. Toutefois, des leaders doivent aussi se positionner et soutenir le processus de transformation des communautés. Pour ce faire, un ou des porteurs de l'innovation doivent être identifiés (Amblard et al., 2005 ; Dessler, 2009). Ces porteurs auront une perspective globale des partenaires impliqués, des actions à réaliser et de l'interrelation des différents facteurs (McShane et Benabou, 2008). Pour avoir une réelle influence, ces porteurs devront avoir un leadership reconnu par les autres acteurs. Ces leaders auront plusieurs responsabilités, dont évaluer la situation, mobiliser les acteurs, défendre le changement, négocier, résoudre des problèmes et agir comme médiateurs (Dessler, 2009). Le succès de ce projet d'envergure dépendra, en grande partie, des qualités démontrées par ces leaders et ces porteurs. Un ou des bons leaders devraient être visionnaires, inspirants et empathiques. Ils devraient aussi posséder de bonnes qualités communicationnelles (Dessler, 2009).

En somme, ces éléments ne constituent que trois principaux repères pouvant guider les premières actions. Ils démontrent que le processus de transformation sociétal menant au développement de VIN accessibles est complexe et que le partenariat pourrait constituer un des critères de réussite de l'initiative. En plus de favoriser un partage des connaissances et des ressources, le partenariat optimisera les actions réalisées par les différentes 


\section{Alejandro ROMERO-TORRES, Claude L. NORMAND et Dany LUSSIER-DESROCHERS}

parties prenantes. Ce partenariat permettra de mettre à profit la complémentarité des expertises et de l'utiliser comme un levier pour le développement d'une société inclusive pour les personnes présentant des limitations cognitives.

\section{Conclusion}

La VIN cherche à développer des services municipaux aux citoyens afin d'améliorer leur qualité de vie et de leur offrir un environnement de participation citoyenne. Par contre, les citoyens peuvent subir une double exclusion: numérique et sociale. Plusieurs technologies et services numériques ne s'adaptent pas au contexte, aux besoins et aux compétences de tous les citoyens. Les citoyens n'étant pas capables d'utiliser les services intelligents-numériques ne peuvent pas contribuer à leur communauté, car ils ne peuvent pas partager leurs données, leurs idées ou participer à la gouvernance des services. Ceci s'avère plus marqué pour les personnes ayant une DI. Afin de développer ou d'adapter les services intelligents-numériques pour tous les citoyens, les municipalités doivent renforcer les ressources numériques des citoyens et de leur environnement. Les villes doivent s'efforcer de rencontrer ou de réduire les cinq exigences d'accessibilité numérique, soit l'accès aux TIC, les exigences sensori-motrices, les exigences cognitives, les exigences techniques et les codes et les conventions sociales. Elles doivent créer des synergies entre les forces et les limites des citoyens avec le soutien de l'environnement afin de surmonter les obstacles à la participation citoyenne. Ainsi, il semble fort pertinent de créer des partenariats pour éviter la fracture numérique et l'exclusion sociale. Toutes les parties prenantes d'une municipalité (élus, fonctionnaires, fournisseurs et citoyens) doivent se pencher sur les obstacles d'accessibilité numérique universelle. Pour ce faire, elles doivent identifier les enjeux auxquels elles sont confrontées, développer une vision commune et définir les mécanismes de coordination et les porteurs pour faire profiter tous les citoyens de la VIN.

Bien que le modèle d'engrenage de l'accessibilité au monde numérique et les trois recommandations pour les partenariats 
Rendre une ville intelligente-numérique accessible pour tous : le cas des personnes présentant une déficience intellectuelle

d'accessibilité soient basés sur le contexte et les enjeux des personnes ayant une DI, l'évidence présentée dans cet article est aussi valide pour d'autres groupes de citoyens qui subissent des obstacles d'accessibilité pour la VIN, soit les personnes aînées les personnes ou sans domicile fixe. Les auteurs planifient d'approfondir ces modèles afin de guider les efforts d'accessibilité des municipalités.

\section{Références}

Abilities Expo (2015). Assistive technology showcase: Interact with tomorrow's technology today. Repéré à http://www.abilities.com/houston/pavilions assistive.html [consulté en novembre 2016]

Amblard, H., Bernoux, P., Herreros, G. et Livian, Y.-F. (2005). Une sociologie de la traduction. Dans H. Amblard, P. Bernoux, G. Herreros et Y.-F. Livian (dir.), Les nouvelles approches sociologiques des organisations (p. 127-185). Paris, France : Éditions du Seuil.

AQETA. (2016). Mesure 30810 du MELS. Repéré à http://www.aqetaoutaouais.qc.ca/WP/?page id =787

Brangier, E., Hammes-Adelé, S. et Bastien, J.-M. C. (2010). Analyse critique des approches de l'acceptation des technologies : de l'utilisabilité à la symbiose humaintechnologie-organisation. Revue européenne de psychologie appliquée, 60, 129-146.

Buijs, P. C. M., Boot, E., Shugar, A., Fung, W. L A. et Bassett, A. S. (2016). Internet safety issues for adolescents and adults with intellectual disabilities. Journal of Applied Research In Intellectual Disabilities. doi: 10.1111/jar.12250

Bunning, K., Trapp, E., Seymour, K., Fowler, M. et Rollett, B. (2010). Survey of the linguistic accessibility of websites designed for people with intellectual disability. Journal of Applied Linguistics and Professional Practice, 7(3), 297-316.

Caragliu, A. et Del Bo, C. F. (2015). Smart Specialization

Strategies and Smart Cities: An evidence-based assessment of 


\section{Alejandro ROMERO-TORRES, Claude L. NORMAND et Dany LUSSIER-DESROCHERS}

European Union policies. The Rise of the City: Spatial Dynamics in the Urban Century, 55-84.

Caragliu, A., Del Bo, C. et Nijkamp, P. (2011). Smart cities in Europe. Journal of Urban Technology, 18(2), 65-82.

CEFRIO (2013). Le gouvernement en ligne : des défis à I'horizon. NETendances 2012, 3(4). Repéré à https://cefrio.qc.ca/media/uploader/NETendances4-gouvLR.pdf

Charlotte, C. (2010). Internet safety survey: Who will protect the children?. Berkeley Technology Law Journal, 25(1), 501-527.

Chen, H.-Y. (2010). Online reading comprehension strategies among fifth- and sixth-grade general and special education students. Education Research and Perspectives, 37(2), 79-109.

Chen, Y.-C. et Dimitrova, D. V. (2008). Civic engagement via egovernment portals: Information, transactions, and policy making. Dans D. F. Norris (dir.), E-government Research: Policy and Management (p. 205-229). Hershey, PA : IGI Global.

Cohen, B. (2012). What exactly is a smart city. Co. Exist, 19. Repéré à https://www.fastcompany.com/1680538/what-exactlyis-a-smart-city

Compiègne, I. (2011). La société numérique en question(s). Auxerre, France : Sciences humaines Éditions.

Dagenais, D., Poirier, K. et Quidot, S. (2012). Raconter l'expérience et comprendre les pratiques : l'inclusion numérique des personnes handicapées au Québec. Québec, Canada : Communautique.

Danial-Saad, A., Weiss, P. L. T. et Schreuer, N. (2012). Assessment of computer task performance (ACTP) of children and youth with intellectual and developmental disability. Disability and Rehabilitation: Assistive Technology, 7(6), 450458.

Danielsson, H., Henry, L., Messer, D. et Rönnberg, J. (2012). Strengths and weaknesses in executive functioning in children with intellectual disability. Research in Developmental Disabilities, 33(2), 600-607. 
Rendre une ville intelligente-numérique accessible pour tous : le cas des personnes présentant une déficience intellectuelle

de Lange, M. et de Waal, M. (2013). Owning the city: New media and citizen engagement in urban design. First Monday, 18(11). doi: https://doi.org/10.5210/fm.v18i11.4954

Dessler, G. (2009). La gestion des organisations : principes et tendances au $\mathrm{XXI}^{\mathrm{e}}$ siècle ( $2^{\mathrm{e}}$ éd.). Saint-Laurent, Canada : Éditions du Renouveau pédagogique.

Dowdell, E. B., Burgess, A. W. et Flores, J. R. (2011). Original research: Online social networking patterns among adolescents, young adults, and sexual offenders. The American Journal of Nursing, 111(7), 28-36.

Dube, W. V. et Wilkinson. K. M. (2014). The potential influence of stimulus overselectivity in AAC: Information from eye tracking and behavioral studies of attention with individuals with intellectual disabilities. Augmentative and Alternative Communication, 30(2), 172-185.

Eurostat (2016). Internet access and use statistics - Households and individuals. Repéré à http://ec.europa.eu/eurostat/statisticsexplained/index.php/Internet access and use statistics households_and_individuals\#Internet_activity_by_age_group

Gabrys, J. (2014). Programming environments: Environmentality and citizen sensing in the smart city. Environment and Planning D: Society and Space, 32(1), 30-48.

Gossett, A., Gossett, A., Mirza, M., Barnds, A. K. et Feidt, D. (2009). Beyond access: A case study on the intersection between accessibility, sustainability, and universal design. Disability and Rehabilitation: Assistive Technology, 4(6), 439450.

Groce, N., Kett, M., Lang, R. et Trani, J. F. (2011). Disability and poverty: The need for a more nuanced understanding of implications for development policy and practice. Third World Quarterly, 32(8), 1493-1513. 


\section{Alejandro ROMERO-TORRES, Claude L. NORMAND et Dany LUSSIER-DESROCHERS}

HabiloMédias. (2015). Cybersécurité. Repéré à http://habilomedias.ca/littératie-numérique-et-éducation-auxmédias/enjeux-numériques/cybersécurité

Helsper, E. J. (2012). A corresponding fields model for the links between social and digital exclusion. Communication Theory, 22(4), 403-426.

Holtfreter, K., Reisig, M. D., Pratt, T. C. et Holtfreter, R. E. (2015). Risky remote purchasing and identity theft victimization among older Internet users. Psychology, Crime and Law, 21(7), 681698.

Hoppestad, B. S. (2013). Current perspective regarding adults with intellectual and developmental disabilities accessing computer technology. Disability and Rehabilitation: Assistive Technology, 8(3), 190-194.

Jaeger, P. T. et Bertot, J. C. (2010). Designing, implementing, and evaluating user-centered and citizen-centered e-government. Dans C. G. Reddick (dir.), Citizens and e government: Evaluating policy and management (p. 1-19). Hershey, PA : Information Science Reference.

Jenkins, H. (2006). Convergence culture: Where old and new media collide. New York, NY : New York University Press.

Karreman, J., van der Geest, T. et Buursink, E. (2007). Accessible Website content guidelines for users with intellectual disabilities. Journal of Applied Research in Intellectual Disabilities, 20(6), 510-518.

Kennedy, H., Evans, S. et Thomas, S. (2011). Can the Web be made accessible for people with intellectual disabilities?. Information Society, 27(1), 29-39.

Le Grand Robert. (2016). Vision. Paris, France : Le Robert.

Lee, J. et Lee, H. (2014). Developing and validating a citizencentric typology for smart city services. Government Information Quarterly, 31, S93-S105. 
Rendre une ville intelligente-numérique accessible pour tous : le cas des personnes présentant une déficience intellectuelle

Lussier-Desrochers, D., Caouette, M. et Godin-Tremblay, V. (2016). Démarche exploratoire sur les tablettes numériques en soutien à la suppléance à la communication orale pour les personnes présentant une déficience intellectuelle (DI) ou un trouble du spectre de l'autisme (TSA) (Rapport de recherche soumis au ministère de la Santé et des Services sociaux du Québec).

Lussier-Desrochers, D., Dupont, M.-Ė., Lachapelle, Y. et Leblanc, T. (2011). Étude exploratoire sur l'utilisation de l'Internet par les personnes présentant une déficience intellectuelle. Revue francophone de la déficience intellectuelle, 22, 41-50.

Lussier-Desrochers, D., L. Normand, C., Fecteau, S., Roux, J., Godin-Tremblay, V., Dupont, M.-Ė., Caouette, M., RomeroTorres, A., Viau-Quesnel, C., Lachapelle, Y. et PépinBeauchesne, L. (2016). Modélisation soutenant l'inclusion numérique des personnes présentant une DI ou un TSA. Revue francophone de la déficience intellectuelle, 27, 5-14.

Lussier-Desrochers, D., L. Normand, C., Romero-Torres, A., Lachapelle, Y., Godin-Tremblay, V., Dupont, M.-Ė., Roux, J., Pépin-Beauchesne, L. et Bilodeau, P. (2017). Bridging the digital divide for people with ID. Cyberpsychology: Journal of Psychosocial Research on Cyberspace, 11(1). doi: http://dx.doi.org/10.5817/CP2017-1-1

McShane, S. L. et Benabou, C. (2008). Comportement organisationnel. Comportements humains et organisations dans un environnement complexe. Montréal, Canada : Chenelière McGraw-Hill.

Michel, G., Masson, R. et Sperandio, J-C. (2006). Internet est-il accessible aux personnes ayant des incapacités ?. Handicap et environnement : objets, espaces et territoires accessibles et utilisables par tous..., 15(1), 12-31.

Morphy, E. (2010, 2 avril). What your iPad will really cost you Hope you're sitting down. Tech News World. Repéré à http://www.technewsworld.com/story/69679.html 


\section{Alejandro ROMERO-TORRES, Claude L. NORMAND et Dany LUSSIER-DESROCHERS}

Nam, T. et Pardo, T. A. (2011, juin). Conceptualizing smart city with dimensions of technology, people, and institutions. Dans Proceedings of the $12^{\text {th }}$ Annual International Digital Government Research Conference: Digital Government Innovation in Challenging Times (p. 282-291). ACM.

Newman, R. C. (2009). Computer security: Protecting digital resources. Mississauga, Ontario : Jones et Bartlett Publishers.

Norris, D. F. et Moon, M. J. (2005). Advancing e-government at the grassroots: Tortoise or hare?. Public Administration Review, 65(1), 64-74.

Perkins, E. A. et LaMartin, K. M. (2012). The Internet as social support for older carers of adults with intellectual disabilities. Journal of Policy and Practice in Intellectual Disabilities, 9(1), 53-62.

Perriault, J. (2006). La norme comme instrument d'accès au savoir en ligne. Hermès, La Revue, 45(2), 77-87.

Peterson, R. (2004). Integration strategies and tactics for information technology governance. Dans W. V. Grembergen, Strategies for information technology governance (pp. 37-80). Hershey, PA : Idea Group Publishing.

PEW Research Center (2018). Declining Majority of Online Adults Say the Internet Has Been Good for Society. PEW report. Repéré à http://www.pewinternet.org/2018/04/30/decliningmajority-of-online-adults-say-the-internet-has-been-good-forsociety/

President's Committee for People with Intellectual Disabilities (PCPID). (2015). Leveling the playing field: Improving technology access and design for people with intellectual disabilities. Repéré à http://www.acl.gov/programs/aidd/Programs/PCPID/docs/PCPID -2015-Report-to-President.pdf

Raghavendra, P., Newman, L., Grace, E. et Wood, D. (2013). "I could never do that before": Effectiveness of a tailored Internet support intervention to increase the social participation of youth 
Rendre une ville intelligente-numérique accessible pour tous : le cas des personnes présentant une déficience intellectuelle

with disabilities. Child: Care, Health and Development, 39(4), 552-561.

Rivas-Costas, C., Anido-Rifon, L., Fernandez-Iglesias, M. J., Gomez-Carballa, M. A., Valladares-Rodriguez, S. et SotoBarreiros, R. (2014). An accessible platform for people with disabilities. International Journal of Human-Computer Interaction, 30(6).

Rodriguez-Bolivar, M. P. (2015). Transforming City Governments for Successful Smart Cities. Public Administration. Genève, Suisse : Springer.

Sadoway, D. et Shekhar, S. (2014). (Re) Prioritizing Citizens in Smart Cities Governance: Examples of Smart Citizenship from Urban India. The Journal of Community Informatics, 10(3). Repéré à http://ci-journal.org/index.php/ciej/article/view/1179

Sallafranque St-Louis F. et Normand, C. L. (2017). From solitude to solicitation: How people with an intellectual disability or autism spectrum disorder use Internet. Cyberpsychology: Journal of Psychosocial Research on Cyberspace, 11(1). doi: https://doi.org/10.5817/CP2017-1-7

Schejter, A., Ben Harush, O. R. et Tirosh, N. (2015). Re-theorizing the "digital divide": Identifying dimensions of social exclusion in contemporary media technologies. Proceedings of European Media Policy 2015: New Contexts, New Approach, Helsinki.

Simard, J. (2015). La ville intelligente comme vecteur pour le développement durable : le cas de la ville de Montréal (Mémoire de maîtrise). Université de Sherbrooke.

Stephenson, J. et Limbrick, L. (2015). A review of the use of touchscreen mobile devices by people with developmental disabilities. Journal of Autism and Developmental Disorders, 45, 3777-3791.

Stock, S. E., Davies, D. K., Davies, K. R. et Wehmeyer, M. L. (2006). Evaluation of an application for making palmtop computers accessible to individuals with intellectual disabilities. Journal of Intellectual and Developmental Disability, 31(1), 3946. 


\section{Alejandro ROMERO-TORRES, Claude L. NORMAND et Dany LUSSIER-DESROCHERS}

Toppeta, D. (2010). The smart city vision: How innovation and ICT can build smart, "liveable", sustainable cities (Think! Report 005/2010). Innovation Knowledge Foundation. Repéré à https://intaaivn.org/images/cc/Urbanism/background\%20documents/Toppe ta_Report_005_2010.pdf

United Nations Broadband Commission For Digital Development (2015). Cyber violence against women and girls: A wolrd-wide wake-up call. Repéré à

http://www.unwomen.org/ /media/headquarters/attachments/sectio ns/library/publications/2015/cyber_violence_gender\%20report.p $d f ? v=1 \& d=20150924 T 154259$

Vodoz, L. (2010). Fracture numérique, fracture sociale : aux frontières de l'intégration et de l'exclusion. Sociologies. Repéré à http://sociologies.revues.org/3333

Wei, K. K., Teo, H. H., Chan, H. C. et Tan, B. C. (2011). Conceptualizing and testing a social cognitive model of the digital divide. Information Systems Research, 22(1), 170-187.

Wei, L. et Hindman, D. B. (2011). Does the digital divide matter more? Comparing the effects of new media and old media use on the education-based knowledge gap. Mass Communication and Society, 14(2), 216-235.

Williams, P. et Hennig, C. (2015). Optimising Web site designs for people with learning disabilities. Journal of Research in Special Educational Needs, 15(1), 25-36.

Williams, P. et Nicholas, D. (2006). Testing the usability of information technology applications with learners with special educational needs (SEN). Journal of Research in Special Educational Needs, 6(1), 31-41.

Wong, A. W. K., Chan, C. C. H., Li-Tsang, C. W. P. et Lam, C. S. (2009). Competence of people with intellectual disabilities on using human-computer interface. Research in Developmental Disabilities, 30(1), 107-123. 